\title{
Who should return for an oral glucose tolerance test? A proposed clinical pathway based on retrospective analysis of $\mathbf{3 3 2}$ children
}

https://doi.org/10.1515/jpem-2020-0689

Received December 4, 2020; accepted January 13, 2021;

published online April 19, 2021

\section{Abstract}

Objectives: Fasting plasma glucose or oral glucose tolerance test (OGTT) is the traditional diagnostic tool for type 2 diabetes (T2DM). However, fasting is required and implementation in all overweight/obese subjects is not practical. This study aimed to formulate a clinical pathway to stratify subjects according to their risk of abnormal OGTT.

Methods: This retrospective study included patients with overweight or obesity who had undergone OGTT in a tertiary paediatric unit from 2012 to 2018. The optimal haemoglobin A1c (HbA1c) cutoff that predicts abnormal OGTT was evaluated. Other non-fasting parameters, in combination with this HbA1c cutoff, were also explored as predictors of abnormal OGTT.

Results: Three hundred and thirty-two patients (boys: 54.2\%, Chinese: 97.3\%) were included for analysis, of which, 272 (81.9\%) patients had normal OGTT while 60 (18.0\%) patients had abnormal OGTT (prediabetes or T2DM). Optimal HbA1c predicting abnormal OGTT was $5.5 \%$ (AUC 0.71 ; sensitivity of $66.7 \%$ and specificity of $71 \%$ ). When $\mathrm{HbA} 1 \mathrm{c} \geq 5.5 \%$ was combined with positive family history and abnormal alanine transaminase (ALT) level,

*Corresponding author: Joanna Yuet Ling Tung, Department of Paediatrics and Adolescent Medicine, LKS Faculty of Medicine, Queen Mary Hospital, The University of Hong Kong, NCB 115, Queen Mary Hospital, 102 Pokfulam Road, Hong Kong, Hong Kong; and Department of Paediatrics, Hong Kong Children's Hospital, Hong Kong, Hong Kong, Phone: +852 2255 4482, Fax: +852 2255 4089,

E-mail: tyl404@ha.org.hk

Sarah Wing-Yiu Poon, Wilfred Hing-Sang Wong, Anita Man-Ching Tsang and Grace Wing-Kit Poon, Department of Paediatrics and Adolescent Medicine, LKS Faculty of Medicine, Queen Mary Hospital, The University of Hong Kong, Hong Kong, Hong Kong. https://orcid.org/0000-0003-0818-9699 (S.W. Poon) the positive predictive value for abnormal OGTT was increased from 33.6 to $61.6 \%$.

Conclusions: HbA1c, family history of T2DM and ALT level could be used to derive a clinical pathway to stratify children who have high risk of abnormal OGTT.

Keywords: obesity; oral glucose tolerance test; paediatrics; type 2 diabetes mellitus.

\section{Introduction}

Childhood obesity is one of the biggest public health challenges in the 21st century and the prevalence has increased at an alarming rate. From 1975 to 2016, the number of obese 5-19 year olds rose more than tenfold globally from 11 million to 124 million [1]. It is associated with increased burden of chronic, non-communicable diseases, one of which being type 2 diabetes (T2DM).

Given the fact that many adolescents with T2DM have minimal symptoms, early detection by screening and timely intervention is essential to prevent diabetic related complication in early adulthood. The American Diabetes Association (ADA) guidelines recommend that overweight and obese children with $\mathrm{BMI} \geq 85$ th percentile for age and sex and additional risk factors should be screened for diabetes every two years, starting at age 10 years or at onset of puberty. These risk factors include maternal history of diabetes or gestational diabetes, family history of T2DM, ethnicity (Native American, African American, Latino, Asian American, Pacific Islander), signs of insulin resistance or conditions associated with insulin resistance. Either a fasting plasma glucose (FPG) or a 2-h oral glucose tolerance test (OGTT) should be performed [2]. Nevertheless, the recommendations on high-risk ethnic group only include Americans, and there are no recommendations for other races. Also, it was found that only a minority of paediatric providers perform FPG or OGTT according to the ADA guidelines [3]. Primary barrier to performing FPG or OGTT in asymptomatic patients is the requirement of fasting, and thus the need for another scheduled visit. For OGTT, at least two blood draws (0 min and $120 \mathrm{~min}$ ) would 
be needed, making the test inconvenient and labourintensive. These barriers may lead to lower testing rate and possibly under-diagnosis [4]. This highlighted that screening tests must be practical and convenient to be implemented effectively. In a busy clinic setting with increasing number of referrals for children with obesity, it seems impractical to test every child affected by overweight/obese with 'risk factors' suggested by ADA with another scheduled visit. A simple clinical pathway to stratify those at higher risk of having abnormal OGTT results from the lower risk ones is needed. This can avoid excessive testing and lessen the burden of additional medical visits for those who have lower chance of abnormal OGTT and allow medical resources to be utilised more efficiently.

Haemoglobin A1c (HbA1c) could possibly be one of the predictors of OGTT results among children with obesity. It is a non-enzymatic product of chronic exposure of haemoglobin to blood glucose which reflects the average plasma glucose concentration over the normal 90- to 120-day average life span of erythyrocytes. With increasing adherence to National Glycohaemoglobin Standardization Program (NGSP), as well as epidemiologic data supporting a relationship between HbA1c and the risk of retinopathy, ADA included HbA1c of $6.5 \%$ or greater and $5.7 \%$ or greater as diagnostic criteria for diabetes and prediabetes respectively in 2010 [5]. While several paediatric studies demonstrated poor sensitivity of HbA1c in diagnosing diabetes with the ADA recommended cutoff [6, 7], HbA1c might still be a useful test for risk stratifications. An important benefit of $\mathrm{HbA1c}$ testing is that it eliminates the need for fasting, and that patients can be tested at any time of the day. Testing HbA1c thus evades the logistic challenge of arranging a return visit. Compared to PG, which is another commonly evaluated parameter in diabetes screening, HbA1c has less biological variability and less fluctuation according to circadian rhythm. It is also not acutely altered by stress levels, or short-term use of common drugs that alter glucose metabolism [8].

The objective of this study is to identify simple nonfasting parameters that can be used to predict abnormal OGTT results in a paediatric obesity clinic. Use of these parameters could help to stratify patients with high risk of abnormal OGTT and reduce the burden of ordering an OGTT for every patient. This could help to formulate a clinical pathway for overweight/obese paediatric patients attending the clinic and allow resources to be utilized more efficiently.

\section{Materials and methods}

\section{Subjects}

A retrospective chart review on paediatric patients with OGTT done in the Department of Paediatrics and Adolescent Medicine, Queen Mary Hospital, The Univeristy of Hong Kong, Hong Kong, a tertiary, University-affiliated paediatric unit, from January 2012 to December 2018 was performed. Since all OGTT were done in the paediatric day centre, the list of patients was retrieved from the admission records of the day centre during that period. These patients were mainly referred from the paediatric obesity clinics or endocrine clinics when they were evaluated for overweight or obesity. In our unit, OGTT would usually be ordered if the body mass index (BMI) centile was $\geq 95$ th centile according to the local growth chart. OGTT would also be ordered in patients with overweight and additional risk factors, e.g. strong family history of T2DM or presence of Acanthosis nigricans.

All overweight/obese patients aged $\leq 20$ years with OGTT and HbA1c tests performed were included. Overweight and obesity were defined using BMI z-score according to the World Health Organization age and gender-specific reference data, with overweight defined as $>+1 \mathrm{SD}$ above the mean (or $\mathrm{BMI} \geq 25 \mathrm{~kg} / \mathrm{m}^{2}$ at $\geq 19$ years) and obesity defined as $>+2$ SD above the mean (or $\mathrm{BMI} \geq 30 \mathrm{~kg} / \mathrm{m}^{2}$ at $\geq 19$ years) [9].

Patients with known genetic syndromes (e.g. Prader-Willi syndrome, Russell-Silver Syndrome), underlying diagnosis other than weight problem that might interfere with glucose homeostasis (e.g. use of drugs that affect glucose metabolism, thalassemia major, cancer survivors, cystic fibrosis etc.) were excluded from analysis. Patients with symptoms suggestive of diabetes (including polyuria, polydipsia or unexplained weight loss) and those who were diagnosed with T2DM and receiving anti-diabetic agent or insulin were also excluded from the study. In addition, since haemoglobin variants and iron deficiency could potentially affect $\mathrm{HbA1c}$ measurements, patients with mean corpuscular volume $(\mathrm{MCV})<80 \mathrm{fL}$ were excluded from analysis.

Clinical information, including gender, family history of T2DM (in first and second degree relatives), anthropometric parameters and biochemical profiles were retrieved from the medical charts.

All patients underwent a standard OGTT $(1.75 \mathrm{~g} / \mathrm{kg}$ of anhydrous glucose solution, maximum $75 \mathrm{~g}$ ) after an 8-h fast. Blood samples for PG and insulin were obtained at $0 \mathrm{~min}, 30$ and $120 \mathrm{~min}$. Normal OGTT was defined based on ADA criteria for glucose tolerance (NGT) with fasting $\mathrm{PG}<5.6 \mathrm{mmol} / \mathrm{L}$ and $2-\mathrm{h} \mathrm{PG}<7.8 \mathrm{mmol} / \mathrm{L}$; impaired fasting glucose (IFG) was defined as fasting PG between 5.6 and $6.9 \mathrm{mmol} / \mathrm{L}$, while impaired glucose tolerance (IGT) was defined as 2-h PG level between 7.8 and $11.0 \mathrm{mmol} / \mathrm{L}$. Patients with IFG and IGT were defined to have prediabetes range of glycaemic response. Those with fasting $\mathrm{PG}$ of $\geq 7.0 \mathrm{mmol} / \mathrm{L}$ or $2-\mathrm{h} \mathrm{PG}$ of $\geq 11.1 \mathrm{mmol} / \mathrm{L}$ were defined to have diabetes range of glycaemic response [5].

\section{Measurements}

PG was measured using the glucose oxidase method and HbA1c analysis was performed by an assay certified by the National Glycohaemoglobin Standardization Program. Homeostatic Model Assessment of Insulin Resistance (HOMA-IR), a validated index for 
measurement of insulin resistance, was calculated according to standard formula: HOMA-IR=fasting insulin $(\mathrm{mIU} / \mathrm{L}) \times$ fasting plasma glucose $(\mathrm{mmol} / \mathrm{L}) / 22.5$. Homeostatic Model Assessment of beta-cell function (\%B), an estimate of steady state beta-cell function, was calculated as: $[20 \times$ fasting insulin $(\mathrm{mIU} / \mathrm{L})] /[$ fasting glucose $(\mathrm{mmol} /$ L)-3.5]; Homeostatic Model Assessment of insulin sensitivity (\%S) was calculated as: 1 HOMA-IR $\times 100$.

Abnormal alanine transaminase (ALT) level was defined based on the age- and gender-specific reference in our local laboratory. The upper limit normal (ULN) for boys aged $\leq 15$ years is $53 \mathrm{U} / \mathrm{L}$ and $35 \mathrm{U} / \mathrm{L}$ for girls. The ULN for boys aged $\geq 16$ years old is $58 \mathrm{U} / \mathrm{L}$ and $36 \mathrm{U} / \mathrm{L}$ for girls.

\section{Statistical analysis}

All statistical analyses were conducted using SPSS 22.2 statistical package (SPSS Inc., Chicago, IL). Continuous variables were expressed as mean \pm standard deviation or median \pm interquartile range (IQR), depending on the normality of the data. Categorical variables were expressed as numbers and percentages. Those with diabetes and prediabetes range of glycaemic response, as defined by OGTT, were considered together as a group (abnormal OGTT group) for statistical analysis. The differences between normal OGTT group and abnormal OGTT group were tested by independent t-test or Pearson chi-square test according to the variable types and data distribution. Receivers operating characteristic (ROC) curves and area under curve were constructed to calculate sensitivity and specificity of $\mathrm{HbA1c}$ cutoffs. The optimal cutoff of HbA1c for the diagnosis of abnormal OGTT was detected by Youden's index. A two-tailed $p$ value $<0.05$ was considered as statistically significant.

\section{Ethics}

The study protocol was reviewed and approved by the Institutional Review Board of the University of Hong Kong/Hospital Authority Hong Kong West Cluster.

\section{Results}

\section{Clinical and biochemical characteristics of patients}

Three hundred and thirty-two patients were included for analysis. Forty (12.0\%) were overweight and $292(88.0 \%)$ were obese. One hundred and fifty two $(45.8 \%)$ were females and 180 (54.2\%) were males. The mean age was $15.4 \pm 2.3$ years and the mean BMI z-score was $2.7 \pm 0.6$. Three hundred and twenty three (97.3\%) patients were Chinese, while nine patients were non-Chinese (2.7\%).

Two hundred and seventy-two patients $(81.9 \%)$ had normal OGTT results, while 60 patients (18.1\%) had abnormal OGTT results - out of which 47 (14.2\%) and $13(3.9 \%)$ had prediabetes and diabetes range of glycaemic response respectively. Table 1 summarizes the demographic, anthropometric and biochemical profile based on their OGTT results. The BMI z-score and age were comparable between the two groups. There were slightly more boys in the normal OGTT group, while the number of boys and girls was exactly the same in the abnormal OGTT group. Patients with abnormal OGTT were more likely to have a family history of T2DM (80.0\%) compared to those with normal OGTT (53.0\%).

While fasting PG, insulin levels and HOMA-IR were higher in the group with abnormal OGTT, the difference was not statistically significant. The mean $\mathrm{HbA1c}$ level was significantly higher in the abnormal OGTT group than normal OGTT group (5.6 vs. 5.3\%, p<0.001). Other biochemical parameters were comparable between the two groups, except there was significantly higher serum ALT level in the abnormal OGTT group ( $36 \pm 32 \mathrm{U} / \mathrm{L})$ compared to normal OGTT group $(26 \pm 20 \mathrm{U} / \mathrm{L})$.

Table 1: Clinical and biochemical characteristics of subjects in the study.

\begin{tabular}{|c|c|c|c|}
\hline & $\begin{array}{r}\text { Normal OGTT } \\
(n=272,81.9 \%)\end{array}$ & $\begin{array}{r}\text { Abnormal OGTT } \\
(n=60,18.1 \%)\end{array}$ & p-Value \\
\hline Age, years & $15.3 \pm 2.2$ & $15.8 \pm 2.1$ & 0.109 \\
\hline Gender (boys) & $150(55.1 \%)$ & $30(50.0 \%)$ & 0.478 \\
\hline BMI SDS & $2.69 \pm 0.58$ & $2.68 \pm 0.57$ & 0.904 \\
\hline $\begin{array}{l}\text { Family history of } \\
\text { diabetes }\end{array}$ & $145(53.0 \%)$ & $48(80.0 \%)$ & $<0.001$ \\
\hline $\begin{array}{l}\text { Fasting } \\
\text { glucose, } \mathrm{mmol} / \mathrm{L}\end{array}$ & $4.7 \pm 2.5$ & $4.9 \pm 0.7$ & 0.540 \\
\hline $\begin{array}{l}\text { Fasting insulin, } \\
\mathrm{mIU} / \mathrm{L}\end{array}$ & $26.3 \pm 25.0$ & $30.3 \pm 19.5$ & 0.246 \\
\hline $\begin{array}{l}30 \mathrm{~min} \\
\text { glucose, } \mathrm{mmol} / \mathrm{L}\end{array}$ & $7.9 \pm 1.3$ & $9.6 \pm 1.9$ & 0.001 \\
\hline $\begin{array}{l}30 \mathrm{~min} \text { insulin, } \\
\mathrm{mIU} / \mathrm{L}\end{array}$ & $214.0 \pm 153.3$ & $200.9 \pm 138.9$ & 0.543 \\
\hline HOMA-IR & $5.4 \pm 5.4$ & $6.7 \pm 4.5$ & 0.080 \\
\hline HOMA-b, \%B & $611.9 \pm 764.6$ & $579.2 \pm 529.9$ & 0.753 \\
\hline HOMA-S, \%S & $27.2 \pm 16.6$ & $21.6 \pm 12.5$ & 0.014 \\
\hline $\mathrm{HbA} 1 \mathrm{c}, \%$ & $5.3 \pm 0.3$ & $5.6 \pm 0.5$ & $<0.001$ \\
\hline AST, U/L & $32.9 \pm 28.8$ & $41 \pm 32.1$ & 0.054 \\
\hline $\mathrm{ALT}, \mathrm{U} / \mathrm{L}$ & $26.0 \pm 20.0$ & $36.0 \pm 32.0$ & 0.008 \\
\hline $\begin{array}{l}\text { Total choles- } \\
\text { terol, } \mathrm{mmol} / \mathrm{L}\end{array}$ & $4.1 \pm 0.7$ & $4.2 \pm 0.9$ & 0.343 \\
\hline $\mathrm{HDL}-\mathrm{C}, \mathrm{mmol} / \mathrm{L}$ & $1.1 \pm 0.2$ & $1.1 \pm 0.2$ & 1.0 \\
\hline LDL-C, $\mathrm{mmol} / \mathrm{L}$ & $2.5 \pm 0.6$ & $2.5 \pm 0.7$ & 1.0 \\
\hline Triglyceride, $\mathrm{mmol} / \mathrm{L}$ & $1.2 \pm 0.6$ & $1.3 \pm 0.6$ & 0.243 \\
\hline
\end{tabular}

BMI SDS, body mass index standard deviation score; HbA1c, haemoglobin A1c; HOMA-IR/HOMA-b/HOMA-S, homeostatic model assessment of insulin resistance, beta cell function, sensitivity; IGI, insulinogenic index; AST, aspartate transaminase; ALT, alanine transaminase; HDL-C, high-density lipoprotein cholesterol; LDL-C, low-density lipoprotein cholesterol. 


\section{HbA1c as predictor of abnormal OGTT}

Figure 1 displays the ROC curve at various cutoffs of $\mathrm{HbA1c}$ for predicting abnormal response in OGTT. The optimal cutoff of HbA1c in identifying abnormal OGTT was 5.5\%, with a sensitivity of $66.7 \%$ and specificity of $71 \%$. The positive predictive value (PPV) at this cutoff was $33.6 \%$ (Table 2).

\section{Combination of parameters to predict abnormal OGTT}

When combining the HbA1c cutoff of 5.5\% with positive family history of diabetes or/and abnormal sex- and agespecific ALT level, the power of predicting abnormal OGTT could be increased. The PPV of abnormal OGTT increased from 33.6 to $50.0 \%$ when this HbA1c cutoff was combined with positive family history. When $\mathrm{HbA1} \geq 55.5 \%$ was combined with both positive family history and abnormal ALT level, the PPV further increased to $61.6 \%$.

\section{Discussion}

To the best of our knowledge, this is among the first studies to examine the use of a combination of clinical and nonfasting parameters in stratifying the low- and high-risk group among a cohort of paediatric patients with

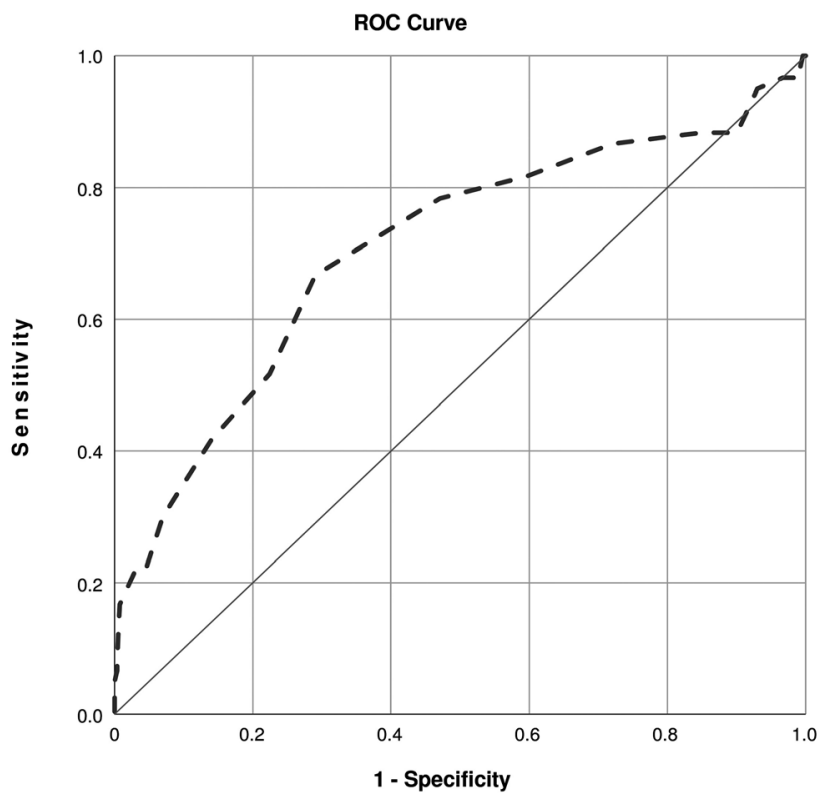

Figure 1: ROC curve at various cutoffs of HbA1c for predicting abnormal response in OGTT.
Table 2: Sensitivities, specificities, positive predictive value (PPV) and negative predictive value (NPV) of different HbA1c cutoff for predicting abnormal OGTT (prediabetes or diabetes).

\begin{tabular}{lrrrr}
\hline HbA1c, \% & Sensitivity, \% & Specificity, \% & PPV, \% & NPV, \% \\
\hline 5.2 & 86.7 & 27.9 & 21.0 & 90.5 \\
5.3 & 81.7 & 40.4 & 23.2 & 90.9 \\
5.4 & 78.3 & 52.9 & 26.8 & 91.7 \\
5.5 & 66.7 & 71.0 & 33.6 & 90.6 \\
5.6 & 51.7 & 77.6 & 33.7 & 87.9 \\
5.7 & 41.7 & 86.0 & 39.7 & 87.0 \\
5.8 & 30.0 & 93.0 & 48.6 & 85.8 \\
5.9 & 21.7 & 95.6 & 52.0 & 84.7 \\
6.0 & 21.7 & 97.1 & 61.9 & 84.9 \\
\hline
\end{tabular}

The "bold" values are the most optimal HbA1c cutoff derived from this study.

overweight or obesity. Since T2DM in adolescence is mostly asymptomatic at diagnosis, and prediabetes is regarded as high-risk state for T2DM [10], this group of young individuals could potentially be exposed to years of hyperglycaemia and it is important to identify them for early interventions. In our cohort, over $97 \%$ were Chinese and close to $60 \%$ had family history of T2DM, thus fulfilling the 'high-risk' group criteria as suggested by ADA to have fasting PG or OGTT screening. Nevertheless, only $18 \%$ of patients had prediabetes or diabetes based on OGTT results. With the global obesity epidemic and thus increasing population of at risk children, a more practical and effective clinical pathway is essential to stratify the higher risk individuals to go for further diagnostic tests, while those at lower risk of prediabetes/T2DM can avoid unnecessary tests and additional hospital/clinic visits. Ultimately, this might increase the case finding of prediabetes and T2DM and allow opportunity for early intervention. With this in mind, our study aimed to look for simple non-fasting parameters as predictors for abnormal OGTT and derive a clinical pathway to guide the management of these children.

In our cohort, using the ADA criteria for prediabetes with a HbA1c cutoff of $\geq 5.7 \%$ only yielded a sensitivity of $41.7 \%$ and a specificity of $86 \%$ in identifying abnormal OGTT (prediabetes or T2DM), meaning that a substantial proportion of patients with prediabetes or diabetes will be missed. Likewise, a number of paediatric studies have also reported fair discriminatory power of the ADA recommended HbA1c cutoff. In a study involving over 1,000 obese paediatric patients, 240 of the 347 (69\%) cases of prediabetes had $\mathrm{HbA1}<5.7 \%$, thus a large number of cases of prediabetes defined by OGTT could not be identified using the recommended cutoff of 5.7\% [7]. Another study involving 254 overweight or obese children also reported a 
low sensitivity of $32 \%$ and specificity of $74 \%$ with a HbA1c cutoff of $5.7 \%$, echoing the fair discriminatory power of this threshold in identifying high risk individuals in the paediatric population [11]. This underscored that the extrapolation of adult HbA1c cutoff to the paediatric population would likely underestimate the prevalence of prediabetes/ T2DM in children. Nevertheless, the advantage in using HbA1c could not be denied. This could be illustrated by a study in the US, in which the rate of screening adolescents with obesity for T2DM increased from 39 to $47 \%$ after the change in recommendations of using $\mathrm{HbA1c}$ for screening. This had led to twice as many T2DM diagnoses during similar time period [12]. On the other hand, a recent prospective study involving a multi-ethnic cohort of children with obesity had shown that, one of the strongest predictors of 2-h glucose at follow-up was baseline HbA1c [7]. This implied that, despite the relatively low discriminatory power to diagnose T2DM with HbA1c alone in the paediatric population, its performance in stratifying adolescents at high risk of prediabetes and diabetes should not be underrated.

Based on our results, a HbA1c cutoff of $5.5 \%$ offers the best combination of sensitivity (66.7\%) and specificity $(71.0 \%)$ in predicting abnormal OGTT when used alone. This cutoff is similar to what was proposed by another study on obese children and adolescents by Preneet et al. in which HbA1c threshold of $5.6 \%$ was suggested as the optimal cutoff in predicting positive OGTT result, with sensitivity of $83.3 \%$ and specificity of $47.2 \%$ [13]. In fact, HbA1c levels had been reported to be positively correlated with age even in non-diabetic populations [14]. Therefore, lower HbA1c cutoffs have been proposed in various paediatric studies. The proposed optimal HbA1c cutoffs for prediabetes in these studies were summarized in Table $3[7$, 15-19].

We also found that, combining serum ALT levels to the HbA1c cutoff of 5.5\% allows us to identify a subgroup of high risk patients. Non-alcoholic fatty liver disease (NAFLD) is the most frequent cause of chronic liver disorders among obese youth. In the United States, Hispanics, followed by non- Hispanic Whites, have the highest prevalence of NAFLD based on elevated ALT level, while prevalence of NAFLD among African-American is much lower [20]. NAFLD encompasses a spectrum of chronic liver disease, beginning with hepatic steatosis, progressing to non-alcoholic steatohepatitis (NASH) in a significant proportion of patients, and eventually to liver fibrosis and cirrhosis in some [21]. It is thought to be a hepatic manifestation of underlying metabolic dysfunction and shares common pathophysiology with T2DM related to excess calorie intake and insulin resistance, and are associated with dyslipidaemia, cardiovascular disease and obesity [22-24]. It is thus not surprising that hepatic steatosis is present in $25-50 \%$ of adolescents with T2DM [25, 26]. While liver biopsy remains gold standard in diagnosis of NAFLD, a serum ALT level are commonly elevated and is an inexpensive and widely available test for screening and initial evaluation of NAFLD [27]. Moreover, previous study has found association between elevated ALT with rising HbA1c level in overweight and obese adolescents [28, 29]. Hence, our study evaluated if combination of serum ALT and HbA1c levels could help in identifying high risk patients. In

Table 3: Paediatric studies on the evaluation of optimal HbA1c cutoff for prediabetes.

\begin{tabular}{|c|c|c|c|c|c|c|c|}
\hline Cohorts & Population ethnicity & Mean age, years & Sample size & AUC & $\begin{array}{l}\text { Suggested optimal } \\
\text { cut point }\end{array}$ & Sensitivity & Specificity \\
\hline Poon et al. 2020 & $\begin{array}{l}\text { Chinese: } 97.3 \% \\
\text { Others: } 2.7 \%\end{array}$ & $15.4 \pm 2.3$ & 332 & 0.71 & $\begin{array}{l}5.5 \% \text { (for } \\
\text { prediabetes or T2DM) }\end{array}$ & $66.7 \%$ & $71 \%$ \\
\hline Tsay et al. 2010 [15] & $\begin{array}{l}\text { Hispanic: } 58 \% \\
\text { African-American: } 10 \% \\
\text { Caucasian: } 6 \% \\
\text { Asian: } 2 \% \\
\text { Native American: } 1 \% \\
\text { Others: } 1 \% \\
\text { Unrecorded: } 22 \%\end{array}$ & $11.2 \pm 3.8$ & 209 & 0.7484 & $5.5 \%($ for IGT) & $85.7 \%$ & $56.9 \%$ \\
\hline Nowicka et al. 2011 [7] & $\begin{array}{l}\text { Caucasians: } 36 \% \\
\text { African American: } 35 \% \\
\text { Hispanic: } 29 \%\end{array}$ & $13.2 \pm 2.8$ & 1,156 & 0.60 & $5.5 \%$ (for IGT) & $57.0 \%$ & $59.9 \%$ \\
\hline Lee et al. 2012 [16] & Korean & Age range: $4-17$ & 126 & 0.651 & $5.8 \%($ for IGT) & $64.7 \%$ & $61.6 \%$ \\
\hline Mutlu et al. 2013 [17] & Turkish & $13.4 \pm 2.6$ & 106 & $\mathrm{n} / \mathrm{A}$ & $5.5 \%($ for IGT) & $63.0 \%$ & $70.0 \%$ \\
\hline Li et al. 2018 [18] & Chinese & $20.2 \pm 2.9$ & 581 & 0.68 & $5.5 \%$ (for IFG and IGT) & $61.4 \%$ & $68.5 \%$ \\
\hline Nam et al. 2018 [19] & Korean & $13.0 \pm 2.5$ & 390 & 0.795 & $5.8 \%$ (for IFG and IGT) & $64.1 \%$ & $83.8 \%$ \\
\hline
\end{tabular}

AUC, area under curve; IGT, impaired glucose tolerance; IFG, impaired fasting glucose. 
fact, serum ALT was significantly higher in our cohort of patients with abnormal OGTT. Using a HbA1c $\geq 5.5 \%$ alone provides a positive predictive value (PPV) of $33.6 \%$ for abnormal OGTT. Combining HbA1c $\geq 5.5 \%$ and abnormal serum ALT levels, together with family history of T2DM, an easily accessible information from patient, increases the PPV to $61.6 \%$. Based on these parameters, we developed a clinical pathway in the management of adolescents with obesity in a clinic-setting based on PPV cutoffs of $>50 \%$, $10-50 \%$ and $<10 \%$ for stratifications (Figure 2). If the combination of HbA1c, family history of T2DM and ALT yielded a PPV $>50 \%$, OGTT should be done. If PPV is between 10 and 50\%, other risk factors e.g. presence of acanthosis nigricans or other comorbidities like polycystic ovarian syndrome, obstructive sleep apnoea, should be taken into consideration when deciding whether to proceed to OGTT. On the other hand, if PPV is $<10 \%$, OGTT can be skipped. As shown in the pathway, a combination of HbA1c $<5.5$ and normal ALT level, with either positive or negative family history of T2DM, yielded PPV of 8.9 and $4.2 \%$ respectively. The low PPVs for abnormal OGTT in these two groups of patients imply that they could be monitored in the clinic without proceeding to further OGTT. With this clinical pathway, 171 (51.5\% of all tests; normal OGTT $=93.6 \%$ ) OGTTs could have been avoided in our cohort.

This study has several strengths. We analysed a modest sized population over a 7-year period at our clinic with $18.1 \%$ individuals having abnormal OGTT. Majority of our patients are Chinese. While it is well known that Asians are more prone to increased insulin resistance at a lower level of adiposity and younger age of onset of diabetes [30], there is lack of literature on how to stratify paediatric patients with obesity into various risk groups. Our study thus contributed to the current body of evidence regarding how best to screen obese children at risk for prediabetes/ T2DM.

There are some caveats to the present study. Our study was conducted in a mainly Chinese population. Hence, HbA1c cutoff might not be generalised to other population, as ethnic disparities in HbA1c levels has been demonstrated in previous studies. In particular, studies which sought to examine ethnic differences in HbA1c levels showed that American Africans have higher HbA1c levels than whites, Hispanics and Asians after adjustment for factors affecting glycaemia [31, 32]. Hence, HbA1c level derived in our study might not be valid, and a higher level is expected to correlate with risk of prediabetes/T2DM in the blacks. In addition, we only evaluated the combination of family history and ALT with HbA1c in this study. If other simple clinical characteristics, for example presence of hypertension, acanthosis nigricans etc. were included in the clinical pathway, we might be able to better stratify patients according to their risk of abnormal OGTT. Unfortunately, with the retrospective nature of the study, most of this information is missing. Besides, while we identified a significant number of adolescents who are at 'low risk' of having prediabetes/diabetes and do not need to return for an OGTT, fasting blood test is still necessary in view of the high prevalence of hypertriglyceridaemia $(20.8 \%)$ in our cohort of patients.

Finally, it is also worth noting that certain ethnic groups of obese adolescents are disproportionately impacted by both T2DM and NAFLD. Hispanics, AfricanAmericans and Asians are more likely than non- Hispanic whites to develop T2DM, while African- Americans are at

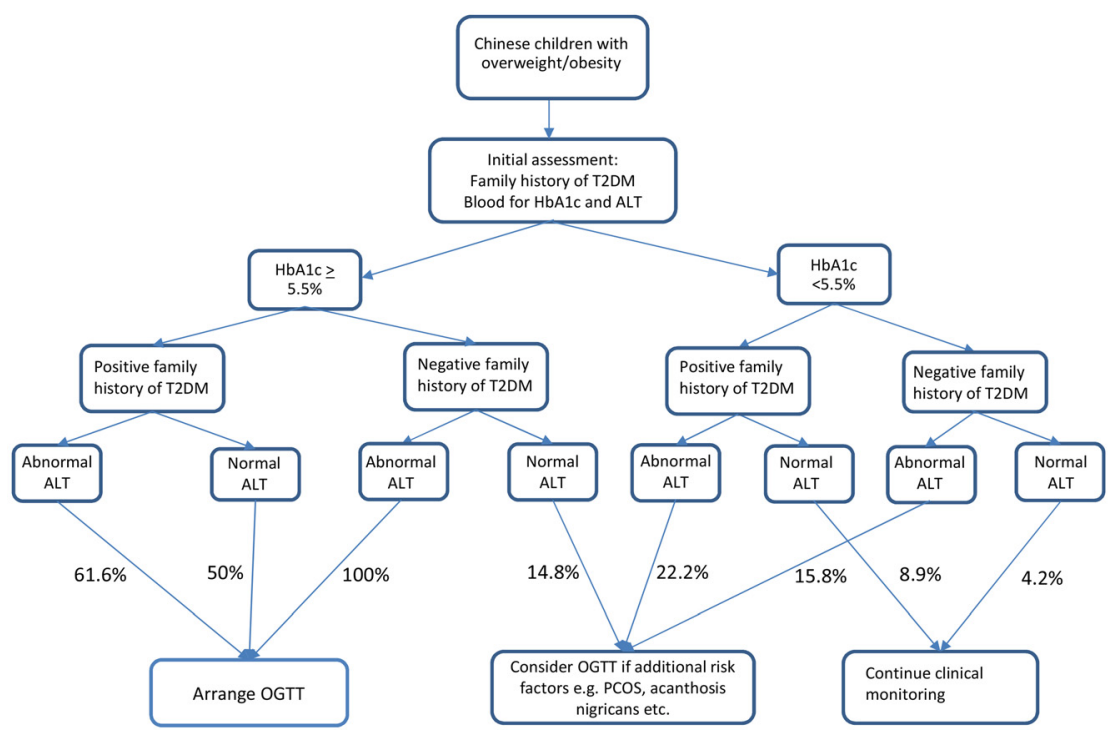

Figure 2: Clinical pathway according to risk of abnormal OGTT based on HbA1c, family history and ALT. 
lowest risk for NAFLD [33, 34]. Hence, combining HbA1c and serum ALT level is a useful tool for Asian, as demonstrated by our study, as well as Hispanic and white adolescents. On the other hand, the utility on obese adolescents of African heritage may be less due to a much lower risk of NAFLD [20].

In conclusion, the use of $\mathrm{HbA1c}$, together with serum ALT level and family history of T2DM represents promising stratifying tools for abnormal OGTT in paediatric patients with overweight or obesity. These tests can be done on the same day of clinic visit without the need of fasting and could exclude a substantial proportion of children from returning for an OGTT. It is a practical strategy to identify children who should be offered further testing with OGTT. Whether using our screening method and HbA1c cutoff would increase the detection rate of prediabetes/diabetes, and ultimately reduce diabetes-related comorbidities in children remains unclear and further longitudinal studies will be required. Future studies are also needed to assess the cost-effectiveness of these screening strategies.

Acknowledgements: The authors would like to thank all the nurses for their help in performing OGTT for our study patients.

Research funding: None declared.

Author contributions: All the authors have accepted responsibility for the entire content of this submitted manuscript and approved submission.

Competing interests: No funding organizations played a role in the study design; in the collection, analysis, and interpretation of data; in the writing of the report; or in the decision to submit the report for publication.

Ethical approval: The study protocol was reviewed and approved by the Institutional Review Board of the University of Hong Kong/Hospital Authority Hong Kong West Cluster.

\section{References}

1. Abarca-Gómez L, Abdeen ZA, Hamid ZA, Abu-Rmeileh NM, Acosta-Cazares B, Acuin C, et al. Worldwide trends in body-mass index, underweight, overweight, and obesity from 1975 to 2016: a pooled analysis of 2416 population-based measurement studies in 128.9 million children, adolescents, and adults. Lancet 2017;390: 2627-42.

2. Association AD. 2. Classification and diagnosis of diabetes: standards of medical care in diabetes-2019. Diabetes Care 2019; 42(1 Suppl):S13-28.

3. Rhodes ET, Finkelstein JA, Marshall R, Allen C, Gillman MW, Ludwig DS. Screening for type 2 diabetes mellitus in children and adolescents: attitudes, barriers, and practices among pediatric clinicians. Ambul Pediatr 2006;6:110-4.
4. Saudek CD, Herman WH, Sacks DB, Bergenstal RM, Edelman D, Davidson MB. A new look at screening and diagnosing diabetes mellitus. J Clin Endocrinol Metab 2008;93:2447-53.

5. American Diabetes Association. Diagnosis and classification of diabetes mellitus. Diabetes Care 2010;33(1 Suppl):S62-9.

6. Lee JM, Wu E-L, Tarini B, Herman WH, Yoon E. Diagnosis of diabetes using hemoglobin A1c: should recommendations in adults be extrapolated to adolescents? J Pediatr 2011;158: 947-52.e3.

7. Nowicka P, Santoro N, Liu H, Lartaud D, Shaw MM, Goldberg R, et al. Utility of hemoglobin A1c for diagnosing prediabetes and diabetes in obese children and adolescents. Diabetes Care 2011; 34:1306-11.

8. Lippi G, Targher G. A laboratory standpoint on the role of hemoglobin A1c for the diagnosis of diabetes in childhood: more doubts than certainties? Pediatric Diabetes 2011;12:183-6.

9. WHO. Obesity and overweight [Internet]. Available from: https:// www.who.int/news-room/fact-sheets/detail/obesity-andoverweight.

10. Tabák AG, Herder C, Rathmann W, Brunner EJ, Kivimäki M. Prediabetes: a high-risk state for developing diabetes. Lancet 2012;379:2279-90.

11. Lee JM, Gebremariam A, Wu E-L, LaRose J, Gurney JG. Evaluation of nonfasting tests to screen for childhood and adolescent dysglycemia. Diabetes Care 2011;34:2597-602.

12. Kapadia C, Zeitler P. Hemoglobin A1c measurement for the diagnosis of type 2 diabetes in children. Int J Pediatr Endocrinol 2012;2012:31.

13. Brar PC, Mengwall L, Franklin BH, Fierman AH. Screening obese children and adolescents for prediabetes and/or type 2 diabetes in pediatric practices: a validation study. Clin Pediatr 2014;53: 771-6.

14. Pani LN, Korenda L, Meigs JB, Driver C, Chamany S, Fox CS, et al. Effect of aging on $\mathrm{A} 1 \mathrm{C}$ levels in individuals without diabetes: evidence from the Framingham offspring study and the national health and nutrition examination survey 2001-2004. Diabetes Care 2008;31:1991-6.

15. Tsay J, Pomeranz C, Hassoun A, Zandieh SO, Rutledge J, Vogiatzi MG, et al. Screening markers of impaired glucose tolerance in the obese pediatric population. HRP 2010;73:102-7.

16. Lee HS, Park HK, Hwang JS. HbA1c and glucose intolerance in obese children and adolescents. Diabet Med 2012;29:e102-5.

17. Mutlu GY, Özsu E, Çizmecioğlu FM, Hatun Ş. Can HbA1c and onehour glucose concentration in standard OGTT be used for evaluation of glucose homeostasis in childhood? J Clin Res Pediatr Endocrinol 2013;5:80-4.

18. Li G, Han L, Wang Y, Zhao Y, Li Y, Fu J, et al. Evaluation of ADA $\mathrm{HbA1C}$ criteria in the diagnosis of pre-diabetes and diabetes in a population of Chinese adolescents and young adults at high risk for diabetes: a cross-sectional study. BMJ Open 2018;8: e020665.

19. Nam HK, Cho WK, Kim JH, Rhie YJ, Chung S, Lee KH, et al. HbA1c cutoff for prediabetes and diabetes based on oral glucose tolerance test in obese children and adolescents. J Kor Med Sci 2018;33:e93.

20. Hudson OD, Nunez M, Shaibi GQ. Ethnicity and elevated liver transaminases among newly diagnosed children with type 2 diabetes. BMC Pediatr 2012;12:174.

21. Angulo P. Nonalcoholic fatty liver disease. N Engl J Med 2002; 346:1221-31. 
22. Fong DG, Nehra V, Lindor KD, Buchman AL. Metabolic and nutritional considerations in nonalcoholic fatty liver. Hepatology 2000;32:3-10.

23. Alisi A, Cianfarani S, Manco M, Agostoni C, Nobili V. Non-alcoholic fatty liver disease and metabolic syndrome in adolescents: pathogenetic role of genetic background and intrauterine environment. Ann Med 2012;44: 29-40.

24. Lawlor DA, Callaway M, Macdonald-Wallis C, Anderson E, Fraser A, Howe LD, et al. Nonalcoholic fatty liver disease, liver fibrosis, and cardiometabolic risk factors in adolescence: a cross-sectional study of 1874 general population adolescents. J Clin Endocrinol Metab 2014;99:E410-7.

25. Newton KP, Hou J, Crimmins NA, Lavine JE, Barlow SE, Xanthakos SA, et al. Prevalence of prediabetes and type 2 diabetes in children with nonalcoholic fatty liver disease. JAMA Pediatr 2016;170:e161971.

26. Nadeau KJ, Klingensmith G, Zeitler P. Type 2 diabetes in children is frequently associated with elevated alanine aminotransferase. J Pediatr Gastroenterol Nutr 2005;41:94-8.

27. Vajro P, Lenta S, Socha P, Dhawan A, McKiernan P, Baumann U, et al. Diagnosis of nonalcoholic fatty liver disease in children and adolescents: position paper of the ESPGHAN
Hepatology Committee. J Pediatr Gastroenterol Nutr 2012;54: 700-13.

28. Strauss RS, Barlow SE, Dietz WH. Prevalence of abnormal serum aminotransferase values in overweight and obese adolescents. J Pediatr 2000;136:727-33.

29. Marchesini G, Brizi M, Morselli-Labate AM, Bianchi G, Bugianesi E, McCullough AJ, et al. Association of nonalcoholic fatty liver disease with insulin resistance. Am J Med 1999;107:450-5.

30. Ramachandran A, Wan Ma RC, Snehalatha C. Diabetes in Asia. Lancet 2010;375:408-18.

31. Herman WH, Ma Y, Uwaifo G, Haffner S, Kahn SE, Horton ES, et al. Differences in $\mathrm{A} 1 \mathrm{C}$ by race and ethnicity among patients with impaired glucose tolerance in the Diabetes Prevention Program. Diabetes Care 2007;30:2453-7.

32. Kamps JL, Hempe JM, Chalew SA. Racial disparity in A1C independent of mean blood glucose in children with type 1 diabetes. Diabetes Care 2010;33:1025-7.

33. Writing Group for the SEARCH for Diabetes in Youth Study Group, Dabelea D, Bell RA, D'Agostino RB, Imperatore G, Johansen JM, et al. Incidence of diabetes in youth in the United States. JAMA 2007;297:2716-24.

34. Pinhas-Hamiel O, Zeitler P. The global spread of type 2 diabetes mellitus in children and adolescents. J Pediatr 2005;146:693-700. 